\title{
Validation of laser-penetration- and electrical-conductivity-based methods of concentration measurement in flow with intense transport of coarse sediment
}

\author{
Jan Krupička ${ }^{1, *}$, Václav Matoušek ${ }^{1}$, Tomáš Picek ${ }^{1}$ and Štěpán Zrostlík ${ }^{1}$ \\ ${ }^{1}$ Faculty of Civil Engineering CTU in Prague, Department of Hydraulics and Hydrology, Thákurova 7, 16000 Prague, Czech Republic
}

\begin{abstract}
Lack of experimentally determined information on inner structure of flows carrying large amount of coarse sediment is an important limitation for development and validation of appropriate mathematical models. Measurement of local flow properties is particularly difficult in case of coarse sediment due to specific features associated with high ratio of the sediment size to the flow depth. This paper focusses on two methods of evaluation of vertical concentration profiles in the flow of a mixture of plastic grains and water in a laboratory flume. The first one was proposed by B. Spinewine et al. in 2011 and it is based on the relation between concentration of solids and the depth of penetration of a laser stripe to the flow, which is evaluated from a high speed camera record. The second method is based on wellknown relation between the concentration and electrical conductivity of a mixture sensed by immersed probe. A sensitivity analysis of both the methods is performed to show the most crucial parameters affecting accuracy of the results. The methods are validated on data measured in fluidization cell with controlled particle concentration. Presented results on flow show limitations and potential of the methods for laboratory studies on liquid-granular flow.
\end{abstract}

\section{Introduction}

High amount of solid material can be carried by flows at high discharges in steep channels with erodible bed. Using laboratory flume to experimentally investigate inner structure of such flows can provide essential information for development and validation of mathematical models based on relations between local concentration, velocity and fluid/solids stresses (e.g. Berzi 2011, Capart \& Fraccarollo 2011, Berzi \& Fraccarollo 2013). Flow conditions in merit of interest can be characterised by presence of layered structure with majority of the solid material transported within a transport layer adjacent to the bed. High solids concentration and predominating effect of interparticle interactions are typical of the transport layer.

To model such conditions physically in the scale of laboratory flumes, relatively light and large particles are often used (Sumer et al., 1996; Capart and Fraccarollo, 2011; Revil-Baudard et al., 2015). A need to measure with a spatial resolution comparable with the particle size, high concentration (implying opacity of mixture) and a wide range of flow velocities make measurement of local concentration difficult. From a variety of measuring methods developed in past (see e.g. Nasr-eldin et al., 1984; Graham et al., 2002; Shook and Daniel, 1965; Revil-Baudard et al., 2015; Capart et al., 2002), we selected two for testing. The first one was recently proposed by Spinewine et al. (2011) for measurement of vertical concentration profiles in laboratory flumes. It is a non-invasive technique based on observation of depth of penetration of laser sheet to the mixture. The second method, on the other hand, implements well known principle based on measurement of electrical conductivity in vicinity of electrodes submerged into the flow.

\section{Principle of selected methods}

\subsection{Laser stripe measurement}

Principle of the method is described in detail in Spinewine et al. (2011). Flow is illuminated at selected cross-section of the flume by a laser sheet penetrating the flow through a transparent side-wall of the flume. The principle is novel and differs from earlier proposed methods based on image processing (Capart et al., 2002; Spinewine et al., 2003). Laser sheet is oriented normal to the flume wall and to the flow direction. At the each vertical position, the particle nearest to the flume wall is hit by the laser.

Depth of laser penetration can be evaluated from a photo of illuminated cross-section taken in direction deviated from the laser sheet (through the side wall) by an angle $\alpha$. Position of a pixel in the photo can be related to a certain position in the illuminated cross-section via a holomorphic transformation. Using the transformation, each photo is ortho-rectified to the coordinate system of

\footnotetext{
*Corresponding author: jan.krupicka@fsv.cvut.cz
} 
flume cross-section. Then a pixel of the maximal brightness, $B_{\max }$, is identified at the each vertical position and its distance from the flume side wall, $y$, is recorded. If the brightness is higher than a certain threshold value $B_{t h}$, this pixel is interpreted as a particle hit by laser and distance $y$ is identified as the depth of laser penetration. The threshold brightness was introduced to filter out unwanted optical effects such as reflections and shadows.

Parameters of the transformation between position of a pixel in the photo and a position in the illuminated cross-section must be calibrated before or after measurement of concentration profile. This is done by taking a calibration photo of a target of known geometry placed at known position in the flume.

As particles enter and leave illuminated crosssection, depth of laser penetration fluctuates in time, but the mean value can be deduced from recorded sequence of photos if observation angle $\alpha$ is known. This mean is related to the concentration: the higher concentration at given vertical position, the higher probability that some particle will be hit by the laser near the wall and the smaller mean depth of laser penetration is observed. Spinewine et al. (2011) derived, under some simplifying assumptions, a mathematical form of this relationship. Hence, a vertical concentration profile can be evaluated from recorded photos of flume cross-section illuminated by a laser sheet.

\subsection{Measurement of local electrical conductivity}

In the simplest configuration, electrical conductivity was evaluated from measurement of voltage and current on pair of electrodes (Ong a Beck, 1975). In configuration proposed by Nasr-El-Din et al. (1987) two pairs of electrodes mounted on vertically shifted gauge were used to evaluate vertical concentration profile in pressurized pipe. Electrical current was set to a pair of field electrodes generating electrical filed and voltage was sensed by a pair of measuring electrodes. This configuration enabled minimization of polarization effects caused by current at surface of field electrodes. Field electrodes were at top and bottom side of cylindrical probe of diameter of $5 \mathrm{~mm}$. Measuring electrodes were at the side of probe in mutual distance of $1 \mathrm{~mm}$. Size of particles in mixture ranged from 0.5 to $5.5 \mathrm{~mm}$. Nasr-El-Din et al used alternating current $1 \mathrm{~mA}$ of high frequency $(1 \mathrm{kHz})$ to further minimize polarization. MacTaggart et al. (1993) used the same frequency and amplitude but slightly different geometrical configuration of electrodes to measure concentration of particles up to $0.5 \mathrm{~mm}$. In CCM probe (Ribberink and Al-Salem, 1995) measuring electrodes are placed in one row between field electrodes in mutual distance of $0.6 \mathrm{~mm}$. The probe was developed to measure concentration of fine sand in oscillatory flow. In all of these applications, particles were relatively small in comparison with characteristic dimensions of flow. The only exception was measurement with 5.5-mm particles, for which Nasr-El-Din et al. (1987) reported considerable distortion of calibration curve for calculation of concentration from measured voltage.
Moreover, MacTaggart et al. (1993) and Nasr-El-Din et al. (1987) pointed out that measurement is affected close to flow boundary.

Principe of measurement is based on relation between electrical conductivity of mixture and concentration of particles. Particles are assumed to be non-conductive. Directly measured quantities are voltage $U$ and current $I$ between electrodes. The ratio $U / I=R_{\text {eq }}$ characterises electrical resistivity of mixture in measuring volume of electrodes. If homogeneous distribution of concentration is assumed in this volume, $R_{\text {eq }}$ depends only on the concentration, conductivity of fluid and geometry of probe. Conductivity of fluid $\sigma_{\mathrm{f}}$ must be measured independently by conductometer to separate its effect $R_{\mathrm{n}}=R_{\mathrm{eq}} \cdot \sigma_{\mathrm{f}}$. Effect of probe geometry is constant for given probe and can be quantified by $R_{\mathrm{n}, \mathrm{pf}}$, i.e. value of $R_{\mathrm{n}}$ calculated from measurement with pure fluid. Then relative conductivity of mixture $\sigma_{\text {rel }}=R_{\mathrm{n}, \mathrm{pf}} / R_{\mathrm{n}}$ depends only on local concentration $c$ : $\sigma_{\text {rel }}=F(c)$. Several forms of function $F$ were suggested in literature (for overview see e.g. MacTaggart et al., 1993). The relation of Rue \& Tobias (1959) has a very simple form of

$$
\sigma_{\mathrm{rel}}=(1-c)^{\mathrm{m}}
$$

\section{Experiment in fluidization cell}

\subsection{Fluidization cell}

Experiment in fluidization cell is suitable way to test method of concentration measurement under control conditions (Spinewine et al., 2011). We performed set of experiments to evaluated potential of selected methods for measurement of concentration in laboratory flume. Fluidization cell $105 \mathrm{~cm}$ high with a rectangular crosssection $22 \mathrm{~cm} \times 20 \mathrm{~cm}$ (Fig. 1).

System of meshes in the bottom section of cell ensured homogeneous inflow of water into the cell. After filling the cell by white plastic particles (rounded, $\mathrm{d}_{50}=3.14 \mathrm{~mm}, \rho=2.36 \mathrm{~kg} / \mathrm{m}^{3}$ ), flow rate thru the cell was gradually increased as long as homogeneous mixture occupying almost entire height of the cell established. This height was measured visually using a scale fixed to the cell wall. Mean concentration in mixture $c_{\mathrm{fc}}$ was calculated as a ratio of volume of solid fraction (calculated from known weight and density of particles) and volume occupied by the mixture. Interface between the mixture and particle-free water above the mixture was most distinct and stable at highest concentration of $46 \%$. At lowest concentration, uncertainty in determination of interface position was approximately $6 \mathrm{~cm}$, i.e. approximately $6.5 \%$ of the measured value. This is a small value in comparison with expected errors of laser stripe measurement and measurement of electrical conductivity. Experiment was performed for seven distinct concentrations between 7 and $46 \%$. Measurement above $46 \%$ was not possible, because homogenous fluidization suddenly switched to regime with convective cells. 


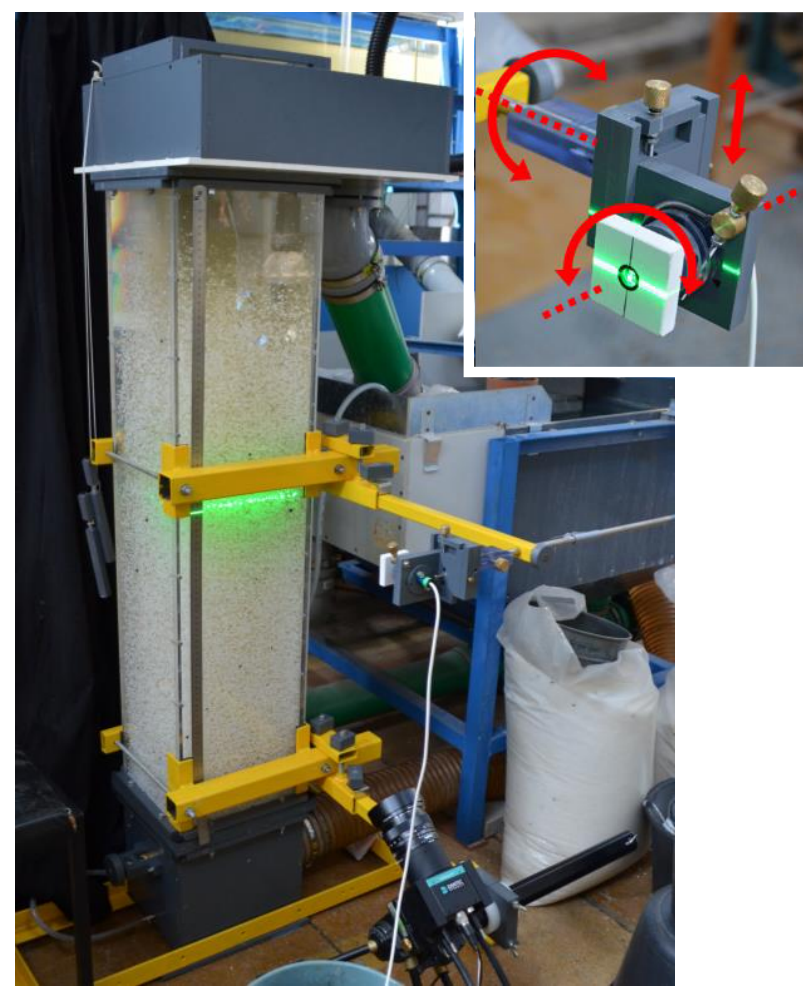

Fig. 1. Fluidization cell with camera and laser mounting. In detail: laser mounting and laser illuminated by reflected laser sheet when adjusting perpendicularity.

\subsection{Laser stripe measurement}

Arrangement of laser and camera enabled evaluation of horizontal concentration profile across the fluidization cell (Fig. 1). We used diode source of green laser sheet (Blau Optoelektronik FP-Mvnano-520-30-30-F, $30 \mathrm{~mW}$, $520 \mathrm{~nm})$. Mounting of the laser enabled adjustment of laser vertical position and inclination. Distance of the laser from the fluidization cell was $34 \mathrm{~cm}$. Perpendicularity of laser sheet and fluidization cell wall was adjusted by employing a mirror attached to the wall - laser inclination was gradually varied as long as reflected laser sheet hit the laser alone (see detail in Fig. 1). High speed camera (Dantec Dynamics FlowSense EO 4M-41 with objective Carl Zeiss Planar 1.4/50 ZF) was used to acquire sequence of photos. Position of an objective of the camera was $39 \mathrm{~cm}$ below the laser and the camera was inclined from horizontal by an angle of about $36^{\circ}$.

Calibration photo was taken when the cell was filled with water but before particles were inserted. Spinewine et all (2011) used a target with the face attached to the wall. Hence, calibration points lied in the plane of wall instead of in the plane of illuminated cross-section, in which depth of laser penetration is evaluated. Then the transformation must be extrapolated to this cross-section. To reduce possible errors resulting from the extrapolation we used different arrangement with a target face normal to the wall as is shown in the figure 2 . When the target was attached to the inner surface of wall, position of laser was carefully adjusted to illuminate edge of the target (the edge divided laser sheet thickness to halves). Then the laser was turned off and the calibration photo was taken. Using described arrangement, transformation is calibrated in the same plane as is that of measurement.

Maximum possible exposition time of $1.6 \mathrm{~ms}$ was chosen for measurement of concentration to achieve high aperture (f/11 and f/16 according actual conditions), and hence high depth of sharpness. Frame rate was set to $58 \mathrm{fps}$ and sequence of 1575 photos (resolution $1168 \times 2352$ ) was acquired at the each concentration.

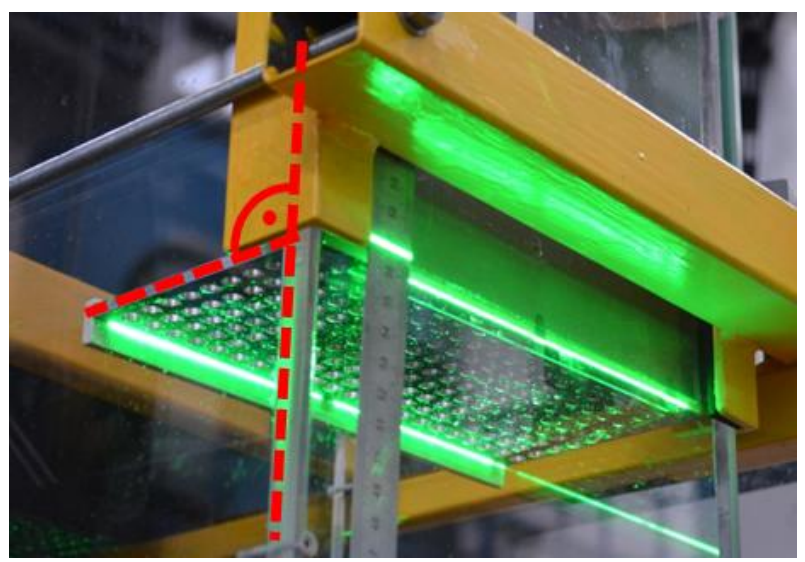

Fig. 2. Calibration target placed in fluidization cell and illuminated by laser sheet.

\subsection{Measurement of local electrical conductivity} Employing numerical simulation of electrical filed generated by electrodes, configuration with pair of measuring electrodes between pair of field electrodes in one line was selected to achieve best spatial resolution (see Fig. 3). Electrodes were oriented against the flow direction to minimize disturbances introduced to the flow in front of the probe. Electrodes were made from stainless steel; the measuring pair has $1.0 \mathrm{~mm}$ and the field pair $1.6 \mathrm{~mm}$ in diameter. Characteristic dimension of measuring volume is given by distance of electrodes, which was chosen to be several particle diameters to minimize effects near particle-probe contacts and influence of particle size on relation between measured voltage and concentration.
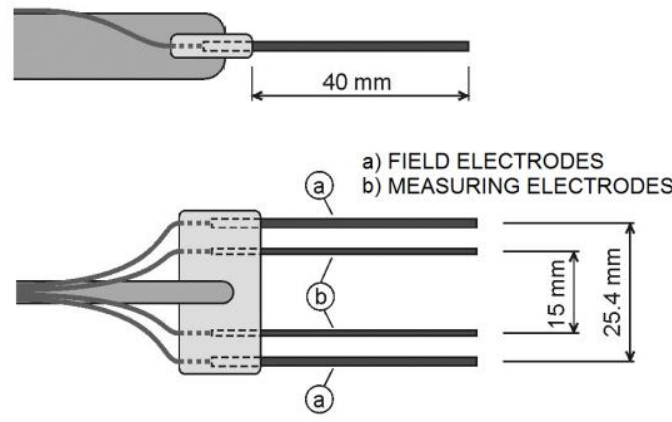

Fig. 3. Probe for measurement of local electrical conductivity.

Direct current was employed instead of highfrequency alternating current recommended by Nasr-ElDin et al. (1987), because the direct one places considerably lower demands on instrumentation. Polarization effects were minimized by using two pairs of electrodes, but not completely eliminated. We found 
that sufficiently accurate measurement with direct current is possible when the voltage at measuring electrodes is measured immediately after the current is supplied to field electrodes. Then the current must be turned off again. Hence, the measurement cannot be continuous. Current of $2 \mathrm{~mA}$ was applied on field electrodes by adjustable current source. Voltage at measuring electrodes was sensed by Analog Devices AD694 transmitter with a voltage input range of 0-2 V. Transmitter output 4-20 mA current signal was led to an A/D convertor via a shielded line. A galvanic separator was inserted to the current loop to prevent closing ground loops.

First, photos for laser stripe measurement were acquired, and then electrical conductivity has been measured at the each concentration. The probe with electrodes has been placed into the fluidization cell to the horizontal position of laser sheet and to the centre of cell cross-section. Numerical simulation of electrical filed generated by electrodes showed that distance from the wall is sufficient to prevent possible effect of wall vicinity reported by MacTaggart et al. (1993). After current was applied to electrodes and voltage was sensed in the mixture, flow through the fluidization cell was stopped and the procedure was repeated in pure fluid to obtain value of $R_{\mathrm{n}, \mathrm{pf}}$. Water conductivity was regularly measured by a conductometer (ExStick EC500).

\section{Results and sensitivity analysis}

\subsection{Laser stripe measurement}

Transformation of position in photo to physical coordinates was calibrated and an observation angle of $\alpha=33.6^{\circ}$ was evaluated. Spinewine at all (2011) didn't proposed any guideline how to determine threshold value $B_{t h}$ of brightness needed to filter out unwanted optical effects. We employed an optimization procedure to find the value of $B_{t h}$ producing minimal mean squared deviation between concentrations evaluated from laser stripe measurement, $c_{\mathrm{lsm}}$, and concentrations in fluidization cell, $c_{\mathrm{fc}}$. Spinewine at al. (2011) found out that calibrated transformation between position in photo and real position in the illuminated cross-section is usually not sufficiently accurate in the near wall region. This causes inaccurate transformation of wall position, and hence evaluated depth of laser penetration to deviates from proper value by a systematic error which has to be corrected. When we included the correction $y_{0}$ to the optimisation as an additional parameter, the mean squared deviation dropped significantly. Figure 4 shows results for the optimised values of $B_{\text {th,opt }}=0.78$ (value of grey at grayscale between $0=$ black and $1=$ white) and $y_{0, \text { opt }}=0.4 \mathrm{~mm}$.

To investigate effect of $B_{t h}$ on evaluated $c_{\text {lsm }}$, we performed sensitivity analysis, results of which are plotted in the figure 5. Each colour indicates experiment at distinct concentration. Values of $c_{\mathrm{fc}}$ are indicated by thin lines and the thick lines show variation of $c_{\mathrm{lsm}}$ with $B_{t h}$. Correction $y_{0}$ is kept on the optimised value. Discrepancies $c_{\mathrm{lsm}}-c_{\mathrm{fc}}$ are indicated by numerical values and by vertical dashed lines at $B_{t h}=B_{\text {th,opt. }}$ All calculations were made at $y_{0}=y_{0, \text { opt }}$. The three curves at lower concentrations show somewhat different course then the other. This is caused by smaller objective aperture used for photos acquired at higher concentrations. Using aperture of $\mathrm{f} / 11$, most of photo pixels representing illuminated practices fell close to the maximal brightness of 1 and smaller brightness can be attributed to other optical effects such as shadows and reflections. Using aperture of $\mathrm{f} / 16$, lower value of $B_{\mathrm{th}}$ is necessary to filter out unwanted optical effects. When higher than necessary $B_{\text {th }}$ is applied, evaluated concentration changes only slightly. Finally, it starts to oscillate at very high $B_{\text {th }}$ becase insufficient amount of relevant data passes through the filter.

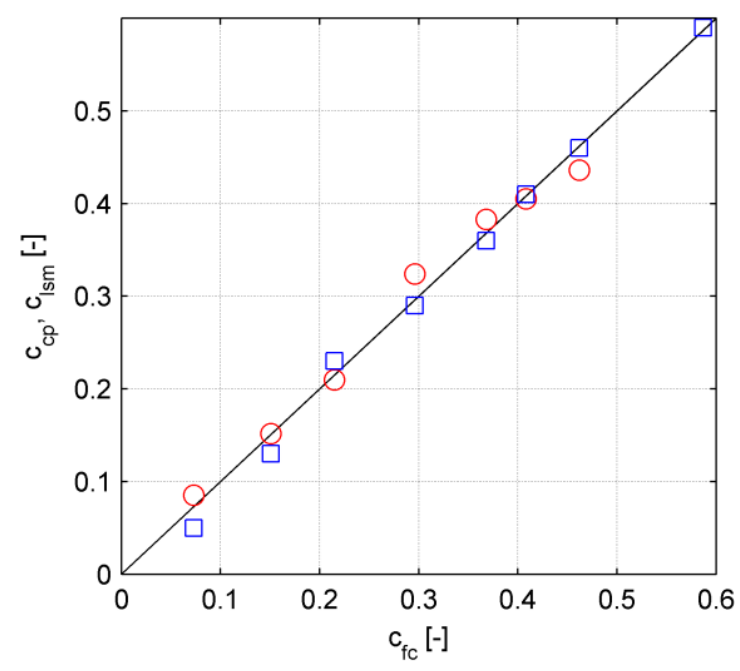

Fig. 4. Evaluated concentrations plotted against the concentrations in fluidization cell: circles - laser stripe measurements, squares - measurement of local conductivity.

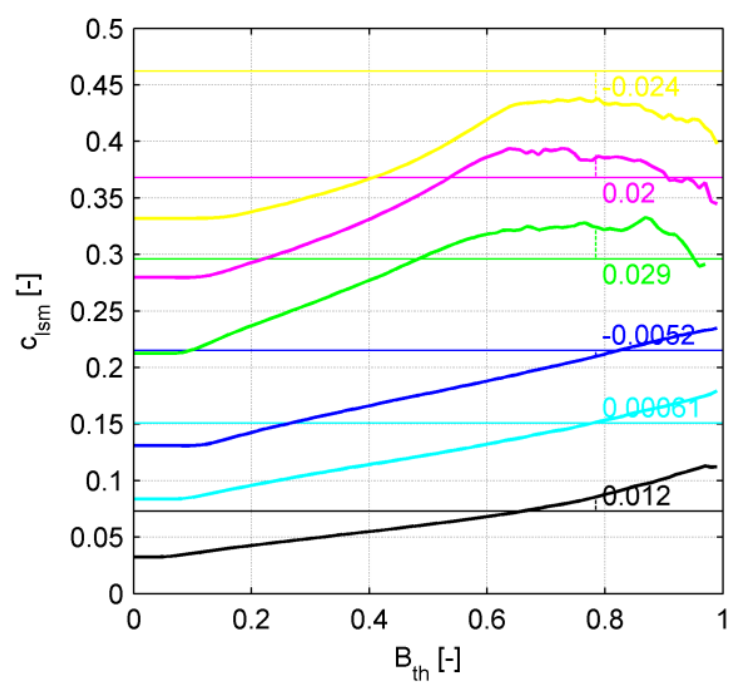

Fig. 5. Effect of threshold brightness on concentration evaluated from laser stripe measurement. Thick line evaluated concentration, thin line - concentration in fluidization cell.

Sensitivity of results to wall position correction $y_{0}$ is shown in the figure 6 . The effect is insignificant at low concentrations, but it becomes crucial as depth of laser 
penetration decreases at high concentration. The correction $y_{0}$ is subtracted from the identified depth of laser penetration $y$. Hence, initial increase of $c_{1 \mathrm{sm}}$ with $y_{0}$ is observed. However, when $y_{0}$ exceeds real position of the wall, data at position $y<y_{0}$ are excluded from analysis. This affects mean value of $y$ and the trend in $c_{\text {lsm }}$ changes.

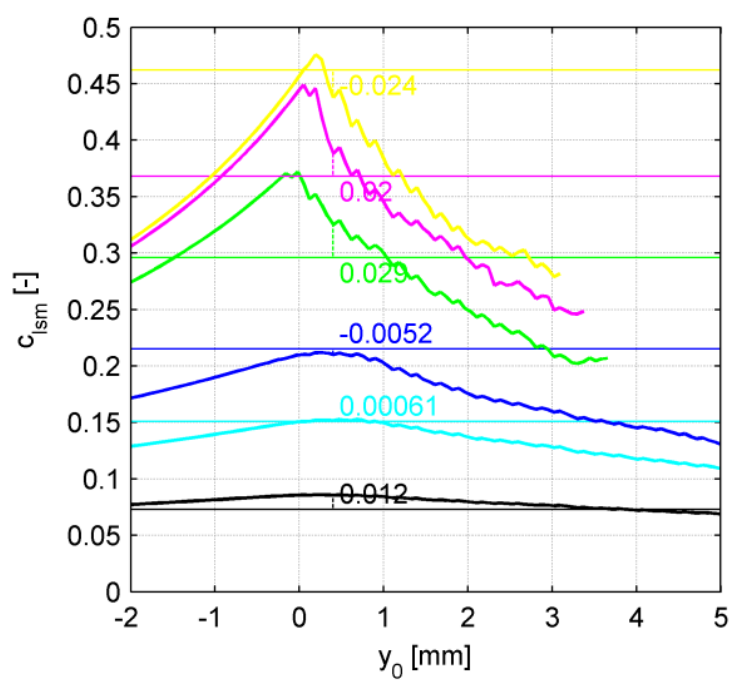

Fig. 6. Effect of wall position correction on concentration evaluated from laser stripe measurement. Thick line evaluated concentration, thin line - concentration in fluidization cell.

Observation angle $\alpha$ is determined from calibrated parameters of holomorphic transformation. This procedure cases that its value is uncertain. Fortunately, figure 7 shows, that evaluated concentration is not very sensitive to errors in $\alpha$ around the value of $30^{\circ}$.

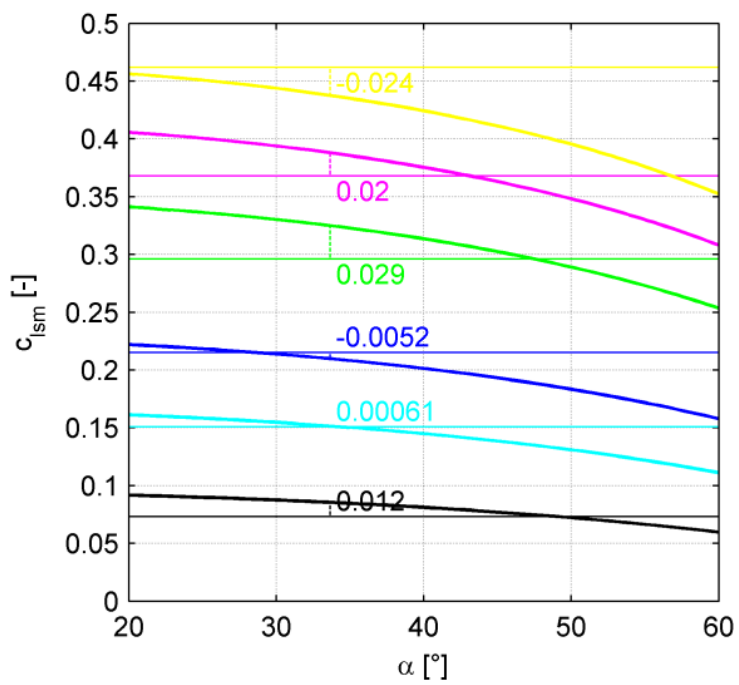

Fig. 7. Effect of observation angle on concentration evaluated from laser stripe measurement. Thick line - evaluated concentration, thin line - concentration in fluidization cell.

\subsection{Measurement of local electrical conductivity} Exponent $m$ in eq. (1) can differ from the value of 1.5 reported by Rue \& Tobias (1959) for spherical particles. It can be easily evaluated from measurement under well- defined conditions of loosely poured particles - we found the value of $1.33 \pm 0.02$ for rounded particles used in our experiments. Comparison of concentrations evaluated from conductivity measurement using $m=1.33$ and mean concentration in fluidization cell is given in figure 4 . The best fit is achieved when the value of $m=1.32$ is instead of 1.33 indicating that the calibration of $m$ is correct. Sensitivity of evaluated concentrations to $m$ varying in the range of $\pm 10 \%$ from the best fitting value is shown in the figure 8 . It is seen that error of $10 \%$ causes

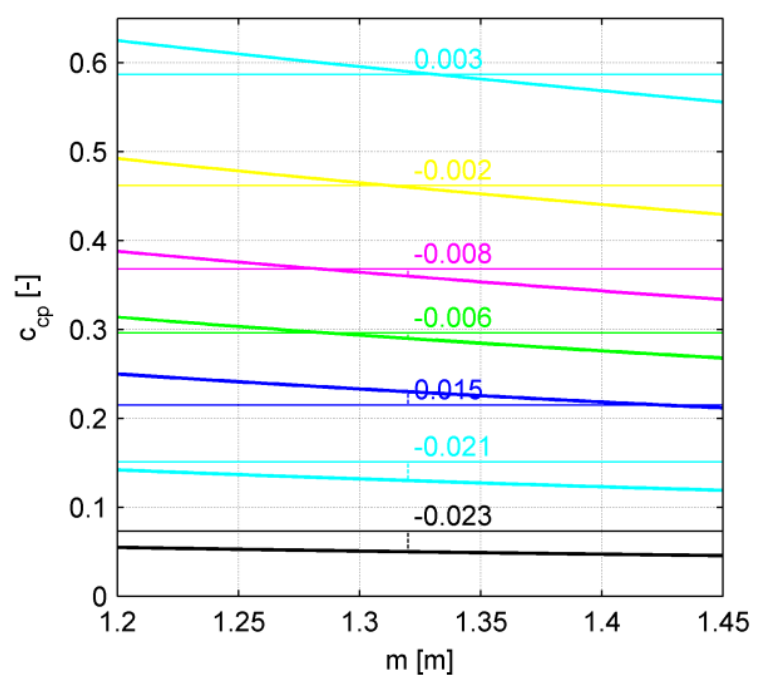

Fig. 8. Effect of exponent in equation (1) on concentration evaluated measurement of electrical conductivity. Thick line evaluated concentration, thin line - concentration in fluidization cell.

A very good match is seen in the figure 8 between $c_{\mathrm{cp}}$ and $c_{\mathrm{fc}}$. However, it should be noted at this point that the method was applied under the ideal conditions of homogeneous mixture and at low velocity up to $13 \mathrm{~cm} / \mathrm{s}$ - the settling velocity of used particles. When employing simple procedure of data evaluation described in section 2.2, spatial resolution of the probe is expected to be insufficient for measurement of local concentrations in transport layer only several grains thick.

\section{Conclusions}

Both tested methods, when properly calibrated, provide accurate values of concentration of particles in homogeneous mixture prepared in fluidization cell.

The method based on measurement of mixture conductivity seems to be more accurate at higher concentrations. It is easy to use from point of view of instrumentation as well as data evaluation. However, in the form tested in this study, its application is limited only to flows with small concentrations gradients.

The method based on laser stripe measurement provides high spatial resolution, and hence it is suitable for application in laboratory flume experiments focused on flow with high concentration gradients. Sensitivity analysis showed high sensitivity of results to parameter $B_{\text {th }}$, for determination of which no general guideline is 
available. Hence, a lack of the method robustness can be considered as its disadvantage.

\section{Acknowledgement}

The research has been supported by the Czech Science Foundation through the grant project No. 16-21421S.

\section{References}

1. D. Berzi, J. Hydraul. Eng., 137(10), 1200-1207, (2011)

2. D. Berzi, L. Fraccarollo, Phys. Fluids, 25, 106601, (2013)

3. H. Capart, L. Fraccarollo, Geophys. Res. Lett., 38, L20402 (2011)

4. B.M. Sumer, A. Kozakiewicz, J. Fredsøe, R. Deigaard, J. Hydraul. Eng. 122, 549-558, (1996)

5. T. Revil-Baudard, J. Chauchat, D. Hurther, P.A. Barraud, J. Fluid Mech., 767 1-30, (2015)

6. H. Nasr-el-din, C.A. Shook, M.N. Esmail, Can. J. Chem. Eng., 62, 179-185, (1984)

7. L. Graham, R. Hamilton, R. Rudman, P. Strode, L. Pullum, Proceedings Hydrotransport 15. Banff, Canada, 149-158, (2002)

8. R.G. Gillies, PhD thesis, University of Saskatchewan, Saskatoon, Canada, (1993)

9. R.E. De La Rue, , C.W. Tobias, , J. Electrochem. Soc., 106, 827-833, (1959)

10. R. S. MacTaggart, H. A. Nasr-El-Din, J. H. Masliyah, Sep. Tech., 3(3), 151-160, (1993)

11. H. Nasr-El-Din, C. A. Shook, J. Colwell,. Int. J. Multiphase Flow, 13(3), 365-378, (1987)

12. K. H. Ong, M. S. Beck, Measurement and Control, 8(11), 453-463, (1975)

13. J. S. Ribberink, A. A. Al-Salem, Coastal Engineering, 25(3-4), 205-225, (1995)

14. B. Spinewine, H. Capart, L. Fraccarollo, M. Larcer, Exp. Fluids 50, 6 (2011)

15. H. Capart, D.L. Young, Y. Zech, Exp. Fluids 32, 2 (2002) 